\title{
Trigger Asynchronies Induced by the Introduction of External Gas Into the Non-Invasive Mechanical Ventilation Circuit: A Bench-to-Bedside Study
}

Cristina Lalmolda ( $\sim$ clalmolda@tauli.cat)

Corporació Sanitaria Parc taulí de Sabadell

\section{Pablo Flórez}

Hospital Universitario Príncipe de Asturias

Carles Grimau

Corporació Sanitaria Parc taulí de Sabadell

Roberto Larrosa

Hospital Universitario 12 De Octubre

Marta Corral

Hospital Universitario 12 De Octubre

Javier Sayas

Hospital Universitario 12 De Octubre

Manel Luján

Corporació Sanitaria Parc taulí de Sabadell

\section{Research Article}

Keywords: Non-invasive Ventilation, trigger asynchronies, autotriggering, ineffective effort, trigger design, bench study, external gas, oxygen therapy, aerosol therapy.

Posted Date: July 6th, 2021

DOl: https://doi.org/10.21203/rs.3.rs-637984/v1

License: (c) (1) This work is licensed under a Creative Commons Attribution 4.0 International License. Read Full License 


\section{Abstract}

Background and objective: Treatments that require the introduction of external gas into the non-invasive ventilation (NIV) circuit, such as aerosol and oxygen therapy, may influence the performance of the ventilator trigger system. The aim of the study was to determine the presence and type of asynchronies induced by external gas in the NIV circuit in a bench model and in a group of patients undergoing chronic NIV.

Methods: Bench study: Four ventilators (one with two different trigger design types) and three gas sources (continuous flow at 4 and $9 \mathrm{l} / \mathrm{min}$ and pulsatile flow at $9 \mathrm{l} / \mathrm{min}$ ) were selected in an active simulator model. The sensitivity of the trigger, the gas introduction position, the ventilatory pattern and the level of effort were also modified.

Clinical study: The same ventilators and gas conditions were used in patients undergoing chronic NIV.

Results: Bench: The introduction of external gas caused asynchronies in $35.9 \%$ of cases (autotriggering $73 \%$, ineffective effort $27 \%)$. Significant differences $(p<0.01)$ were detected according to the ventilator model and the gas source.

Clinical study: In seven patients, the introduction of external gas induced asynchrony in $20.4 \%$ of situations (77\% autotriggering). As in the bench study, there were differences in the occurrence of asynchronies depending on the ventilator model and gas source used.

Conclusion: The introduction of external gas produces alterations in the ventilator trigger. These alterations are variable, and depend on the ventilator design and gas source. This phenomenon makes it advisable to monitor the patient at the start of treatment.

\section{Introduction:}

Non-invasive ventilation (NIV) is widely used in the treatment of acute ${ }^{1}$ and chronic respiratory failure, and is mainly administered for night-time ventilatory support. ${ }^{2}$

NIV can be used in isolation or combined with other treatments, which may require external devices that introduce variable amounts of gas mixture into the circuit. Aerosol medications are commonly used in the treatment of respiratory tract diseases ${ }^{3}$. They offer certain advantages over non-inhalation therapy, one being that they minimize toxicity by providing higher concentrations of the drug in the airway while low systemic concentrations are maintained. Aerosolized treatment is used relatively frequently with NIV, mainly to administer bronchodilator drugs and/or antibiotics. ${ }^{4}$ In an acute situation, the suspension of NIV in order to administer these treatments may entail risks for the patient. ${ }^{3,5}$

The efficacy of aerosol therapy in patients undergoing NIV has been studied in terms of drug administration and also factors regarding the device (i.e., the position and type of nebulizers). ${ }^{3,6-12}$ 
Regarding the implications for the ventilator, it is known that the administration of external gas can influence the patient's tidal volume and patient-ventilator synchronization. ${ }^{13}$ As a consequence, it may be necessary to adjust the ventilator mode and parameters when providing aerosol therapy for patients on mechanical ventilation. $6,14,15$

Another clinical situation that may require the introduction of external gas into the ventilation circuit is the need to provide supplemental oxygen at variable flows, usually lower than the nebulization flow. Many of the ventilators used for NIV are not equipped with an oxygen mixer or specific ports for the administration

of supplemental oxygen; therefore, supplemental oxygen is usually administered by adding it to the mask or the circuit. ${ }^{9}$ This external flow may have an effect on the performance of NIV, especially its trigger function, although this effect has not been studied to date. ${ }^{16}$

The aim of the present study was to evaluate the presence of trigger asynchronies resulting from the introduction of an external gas into the circuit during NIV in two settings:

1. In a bench study, under different ventilatory patterns (obstructive or restrictive), sensitivity levels, levels of patient effort ${ }^{17}$ and different sources, quantities and locations of the gas introduced (continuous versus pulsatile flow compressor)

2. In a short clinical study in patients receiving chronic NIV, in which the bench model previously described is reproduced.

\section{Methods:}

\section{Design of the bench study}

The experiment was performed under simulated conditions in the NIV laboratory. A breathing simulator (series 1101, Hans Rudolph, Inc., Shawnee, Kansas) was used to test the different ventilators, which were connected to the simulator through a single standard 2-m tube with a leak in the expiratory port (Model 5804000 , Intersurgical España SL, Madrid) placed at the distal end of the ventilator. The leak port was maintained throughout the experiment to simulate the intentional leak used in clinical practice in NIV with a single-limb configuration.

For the introduction of the external gas, a T-piece with a 3.5-mm diameter side port (Model 2713000. Intersurgical España SL, Madrid) was placed proximally to the ventilator first and then distally to the leak.

Three different treatment modalities were added to the NIV set-up:

- In the first model, a compressor (Mini Plus, APRES MEDICAL SL, Spain) was connected to a tube in the circuit. The flow of the compressor was monitored prior to placement, and a value of $9 \mathrm{l} / \mathrm{min}$ was obtained (Figure 1).

- The previous model was reproduced in terms of the amount of gas introduced, but a continuous gas source with a pressure reducer and flowmeter (compressed air bottle, Nippon Gases, Madrid) was 
used.

- Finally, in the third model, a continuous gas source was used at 4 litres per minute. Medical air was used to avoid oxidation of the simulator parts due to the use of pure oxygen.

The simulator was programmed as follows: For lung mechanics variables, compliance was set at 30 $\mathrm{ml} / \mathrm{cm} \mathrm{H} 2 \mathrm{O}$ and resistance was set at $5 \mathrm{~cm} \mathrm{H} 2 \mathrm{O} / \mathrm{L} / \mathrm{s}$ in a restrictive pattern and at $60 \mathrm{ml} / \mathrm{cm} \mathrm{H} 20$ and 18 $\mathrm{cm} \mathrm{H2O/L/s} \mathrm{respectively} \mathrm{in} \mathrm{an} \mathrm{obstructive} \mathrm{pattern} \mathrm{(21).} \mathrm{To} \mathrm{simulate} \mathrm{patient} \mathrm{effort,} \mathrm{the} \mathrm{amplitude} \mathrm{was}$ programmed at $6 \mathrm{cmH} 20$ for low effort and $14 \mathrm{cmH} 20$ for high effort, depending on the condition simulated. Finally, the frequency of spontaneous breathing was established at 15 breaths $/ \mathrm{min}$.

Four commercial ventilators (one with two types of triggers) with the same pressure support were compared: (1) Vivo 50 (General Electric, Sweden), (2) Astral 150 (ResMed, Australia), (3) Trilogy 100 (Philips Respironics, Pennsylvania), and (4) Puritan Bennett (PB) 560 (Covidien, Massachusetts). The parameters selected for each ventilator are summarized in Table 1.

Table 1. Parameterization of the ventilators in the bench study model

\begin{tabular}{|c|c|c|c|c|c|c|c|c|c|c|}
\hline \multirow[t]{2}{*}{ Model } & \multirow[t]{2}{*}{ PS } & \multicolumn{2}{|c|}{ Inspiratory trigger } & \multicolumn{3}{|c|}{ Cycle } & \multicolumn{3}{|c|}{ Ramp } & \multirow[t]{2}{*}{$\mathrm{RF}^{\mathrm{X}}$} \\
\hline & & $S^{* *}$ & $M^{\star \star}$ & $N S^{* *}$ & $S^{\rrbracket}$ & $M^{\rrbracket}$ & $\mathrm{L}^{\mathbb{}}$ & ০§ & $\mathrm{R}^{\S}$ & \\
\hline Trilogy & $10 *$ & $2 \mathrm{~lm}$ & $5 \mathrm{~lm}$ & $91 \mathrm{~m}$ & $60 \%$ & $50 \%$ & $30 \%$ & 2 & 3 & 12 \\
\hline $\begin{array}{l}\text { Trilogy } \\
\text { ATrak }\end{array}$ & $10 *$ & \multicolumn{2}{|c|}{ AUTOMATIC } & & \multicolumn{2}{|c|}{ AUTOMATIC } & & 2 & 3 & 12 \\
\hline Vivo 50 & $10 *$ & 2 & 4 & 7 & 7 & $4-5$ & 2 & 2 & 4 & 12 \\
\hline Astral 150 & $10 *$ & High & Medium & low & High & Medium & Low & $\begin{array}{l}150 \\
\mathrm{~ms}\end{array}$ & $\begin{array}{l}250 \\
\mathrm{~ms}\end{array}$ & 12 \\
\hline PB 560 & $10 *$ & 2 & 4 & 6 & $60 \%$ & $50 \%$ & $30 \%$ & 2 & 3 & 12 \\
\hline
\end{tabular}

* IPAP: 15; EPAP: 5, **S: Sensitive; M: Moderately sensitive; NS: Not sensitive, ${ }^{\mathbb{S} S:}$ Short; M: Medium; L: Long, §O: Obstructive, R: Restrictive, ${ }^{\mathrm{X}}$ : Respiratory Frequency

Signal acquisition system: An external polygraph (16Sp Powerlab, AD Instruments, Australia) equipped with two pressure transducers (model 1050) and two pneumotachographs (S300, instrumental dead space $=70 \mathrm{ml}$, resistance $=0.0018 \mathrm{~cm} \mathrm{H} 20 / \mathrm{L} / \mathrm{s}$ ) was used. The sampling frequency was adjusted to 200 $\mathrm{Hz}$, and the polygraph was connected to a personal computer equipped with Chart 7.0 software for Windows.

The ventilation periods were 1 minute (without gas, gas, without gas). This sequence was performed twice in each group of established conditions. The gas-induced asynchronies were compared by interpreting the graphs of pressure and flow time according to the SOMNONIV algorithm ${ }^{18}$. 
The study was conducted with patients from the Pneumology Service of the Corporació Sanitària Parc Taulí (Sabadell, Barcelona) who met the following criteria: age over 18, hospital admission for acute chronic respiratory failure, home NIV (single-limb system with intentional leakage) and more than six months of use with adequate compliance ( 5 hours/night or moe). Patients with underlying psychiatric disease were excluded. The study was conducted during the patient's predischarge phase (the same day or the day before).

Protocol: The procedure was performed in the patient's room during his or her hospital stay to avoid additional visits. The patient was placed in the supine position, and the usual ventilator interface was placed in a single-limb system, along with the commercial ventilator to be studied. The same ventilators tested in the bench model were evaluated at the bedside in random order. The parameters of the ventilator were the same as those that the patients used at home (that is, unlike the bench study, the sensitivity of the trigger was not modified). The monitoring system was essentially the same as previously described, with the incorporation of thoracic and abdominal bands and parasternal electromyography to better evaluate asynchronies, in addition to pulse oximetry control. The gas sources were the same as those used in the bench study model. All methods were performed in accordance with the relevant guidelines and regulations. The study was approved by the hospital ethics committee of “Comité de ética e investigación con medicamentos (CEIm) del Parc Taulí de Sabadell” (ref. 2019/511). Written informed consent was obtained.

\section{Statistical analysis}

Quantitative data are expressed as means and standard deviation ( $\mu, s d)$ according to the normality of the variables, which was assessed using the Shapiro Wilk test. Qualitative data are expressed as absolute frequencies. The chi-square test or Fisher's exact test was used in the absence of normality to evaluate the presence or absence of gas-induced asynchrony by comparing the variables controlled in the bench study and the ventilator model, gas source, and interindividual variability in the clinical study. The level of significance was established at $p<0.05$. Accepting an alpha risk of 0.05 and a beta risk of 0.2 , seven subjects were necessary to recognize a significant difference, consisting in the absence of asynchronies before gas and a high presence after gas, considering a drop-out rate of $20 \%$.

\section{Results:}

Bench study model

A total of 312 simulation sequences were obtained after combining the different variables (gas source, ventilatory pattern, position of entry into the circuit, trigger sensitivity and patient effort). In 67 cases $(21.4 \%)$, asynchrony under basal conditions was documented; these were therefore excluded from the final analysis, which included 245 combinations. 
Table 2 shows the differences between ventilators in terms of percentages and types of baseline asynchronies in the traces that were not included in the analysis.

Table 2

Distribution between ventilators of the simulation sequences in which there was baseline asynchrony $(p<$ 0.01 among ventilators, chi-square test)

\begin{tabular}{|c|c|c|c|c|c|}
\hline & & \multicolumn{3}{|c|}{ Type of basal asynchrony, if present } & \multirow[t]{2}{*}{ Total } \\
\hline & & $\begin{array}{l}\text { Without } \\
\text { asynchrony }\end{array}$ & Autotrigger & $\begin{array}{l}\text { Ineffective } \\
\text { effort }\end{array}$ & \\
\hline \multirow{5}{*}{$\begin{array}{l}\text { Ventilator } \\
\text { model }\end{array}$} & Trilogy & $60(83.3 \%)$ & $12(16.7 \%)$ & 0 & 72 \\
\hline & $\begin{array}{l}\text { Trilogy } \\
\text { AutoTrak }\end{array}$ & $23(95.8 \%)$ & $1(4.2 \%)$ & 0 & 24 \\
\hline & Vivo 50 & $50(69.4 \%)$ & $20(27.7 \%)$ & $2(2.8 \%)$ & 72 \\
\hline & Astral 150 & $70(97.2 \%)$ & $1(1.4 \%)$ & $1(1.4 \%)$ & 72 \\
\hline & PB 560 & $42(58.3 \%)$ & $22(30.6 \%)$ & $8(11.1 \%)$ & 72 \\
\hline \multicolumn{2}{|l|}{ Total } & $245(78.5 \%)$ & $56(17.9 \%)$ & $11(3.5 \%)$ & 312 \\
\hline
\end{tabular}

The introduction of gas induced asynchronies in $35.9 \%(88 / 245)$ of the simulations. Autotrigger was the most frequent asynchrony (64/88), followed by ineffective effort (24/88). In 56/88 cases, the gasinduced asynchrony remained unchanged (without correction) throughout the period of gas introduction. Ineffective effort was corrected in a significantly higher percentage of situations $(16 / 24)$ than autotrigger $(16 / 64, p<0.01)$.

Regarding the factors conditioning asynchrony, statistically significant differences were found in the presence of asynchronies between ventilators, type of gas (pulsatile vs continuous), gas flow (4 vs 9 litres in continuous flow), level of effort of the simulator, ventilatory pattern and trigger sensitivity. Table 3 shows the results, differentiated by the asynchronies induced. 
Table 3

Percentage and type of asynchronies induced by the introduction of external gas as a function of the variables controlled in a bench study model.

\begin{tabular}{|c|c|c|c|c|c|c|}
\hline & & $\begin{array}{l}\text { No } \\
\text { asynchrony }\end{array}$ & Autotriggering & $\begin{array}{l}\text { Ineffective } \\
\text { effort }\end{array}$ & Total & $\begin{array}{l}\text { p } \\
\text { value* }\end{array}$ \\
\hline \multirow[t]{5}{*}{ Ventilator } & Trilogy & $50(83.3 \%)$ & $1(1.7 \%)$ & $9(15 \%)$ & 60 & \multirow[t]{5}{*}{$<0.01$} \\
\hline & $\begin{array}{l}\text { Trilogy } \\
\text { (AutoTrak) }\end{array}$ & $19(82.6 \%)$ & $1(4.3 \%)$ & $3(13.1 \%)$ & $23 \S$ & \\
\hline & Vivo 50 & 24 (48\%) & $22(44 \%)$ & $4(8 \%)$ & 50 & \\
\hline & Astral 150 & $47(67.1 \%)$ & $17(24.3 \%)$ & $6(8.6 \%)$ & 70 & \\
\hline & PB 560 & $17(40.5 \%)$ & $23(54.8 \%)$ & $2(4.7 \%)$ & 42 & \\
\hline \multirow[t]{3}{*}{$\begin{array}{l}\text { Type and } \\
\text { source of gas }\end{array}$} & $\begin{array}{l}\text { Compressor at } 9 \\
\mathrm{~L} / \mathrm{min}\end{array}$ & $30(37.5 \%)$ & $44(55 \%)$ & $6(7.5 \%)$ & 80 & \multirow[t]{3}{*}{$<0.01$} \\
\hline & $\begin{array}{l}\text { Continuous gas } 9 \\
\text { I/min }\end{array}$ & $54(63.6 \%)$ & $15(17.6 \%)$ & $\begin{array}{l}16 \\
(18.8 \%)\end{array}$ & 85 & \\
\hline & $\begin{array}{l}\text { Continuous gas } \\
\text { at } 4 \mathrm{I} / \mathrm{min}\end{array}$ & $73(91.2 \%)$ & $5(6.3 \%)$ & $2(2.5 \%)$ & 80 & \\
\hline \multirow[t]{2}{*}{ Pattern } & Obstructive & $73(58.8 \%)$ & $30(24.2 \%)$ & $21(17 \%)$ & 124 & \multirow[t]{2}{*}{$<0.01$} \\
\hline & Restrictive & $84(69.4 \%)$ & $34(28.1 \%)$ & $3(2.5 \%)$ & 121 & \\
\hline \multirow[t]{2}{*}{ Gas position } & $\begin{array}{l}\text { Proximal to the } \\
\text { ventilator }\end{array}$ & $69(57.5 \%)$ & $39(32.5 \%)$ & $12(10 \%)$ & 120 & \multirow[t]{2}{*}{ ns } \\
\hline & $\begin{array}{l}\text { Distal to the } \\
\text { ventilator }\end{array}$ & $88(70.4 \%)$ & $25(20 \%)$ & $12(9.6 \%)$ & 125 & \\
\hline \multirow{2}{*}{$\begin{array}{l}\text { Effort of } \\
\text { simulator }\end{array}$} & High & $91(74 \%)$ & $31(25.2 \%)$ & $1(0.8 \%)$ & 123 & \multirow[t]{2}{*}{$<0.01$} \\
\hline & Low & $66(54.1 \%)$ & 33 (27\%) & $\begin{array}{l}23 \\
(18.9 \%)\end{array}$ & 122 & \\
\hline \multirow{4}{*}{$\begin{array}{l}\text { Trigger } \\
\text { sensitivity }\end{array}$} & Sensitive & $27(61.4 \%)$ & $15(34.1 \%)$ & $2(4.5 \%)$ & 44 & \multirow[t]{4}{*}{0.01} \\
\hline & Intermediate & $57(62 \%)$ & 29 (31.5\%) & $6(6.5 \%)$ & 92 & \\
\hline & Not very sensitive & $54(62.8 \%)$ & $19(22.1 \%)$ & $\begin{array}{l}13 \\
(15.1 \%)\end{array}$ & 86 & \\
\hline & AutoTrak & $19(82.6 \%)$ & $1(4.3 \%)$ & $3(13.1 \%)$ & $23 \S$ & \\
\hline \multicolumn{7}{|c|}{${ }^{*}$ Chi-square test } \\
\hline
\end{tabular}


After gas closure, asynchronies were induced in 101 situations (96 with autotriggering), and the vast majority (95 in total) were transient. Only in six situations was the autotriggering constant until the end of the phase.

\section{Short Clinical study}

Seven patients were included in the study in the short validation cohort. Their characteristics are detailed in Table 4, which also presents the most relevant ventilator parameters.

Table 4

Characteristics and devices of the patients included in the clinical study

\begin{tabular}{|cllllllll|}
\hline $\begin{array}{l}\text { Num } \\
\text { f }\end{array}$ & $\begin{array}{l}\text { Sex } \\
\text { f }\end{array}$ & $\begin{array}{l}\text { Age } \\
\text { (years) }\end{array}$ & Pathology & PS & Trigger & Cycle & Ventilator & Interphase \\
\hline 1 & M & 70 & COPD & 13 & $\begin{array}{l}\text { Moderately } \\
\text { sensitive }\end{array}$ & Medium & LUMIS & Oronasal \\
\hline 2 & M & 64 & Restrictive & 10 & Sensitive & Medium & LUMIS & Oronasal \\
\hline 3 & F & 61 & Neuromuscular & 15 & Sensitive & Long & ASTRAL & Oronasal \\
\hline 4 & M & 78 & COPD & 12 & $\begin{array}{l}\text { Moderately } \\
\text { sensitive }\end{array}$ & Short & LUMIS & Oronasal \\
\hline 6 & M & 64 & COPD & 12 & Sensitive & Short & TRILOGY & Oronasal \\
\hline 7 & M & 65 & Restrictive & 12 & $\begin{array}{l}\text { Moderately } \\
\text { sensitive }\end{array}$ & Long & STELLAR & Oronasal \\
\hline
\end{tabular}

After the introduction of external gas, new-onset asynchronies occurred in a total of 22/105 situations (20.4\%).

The most prevalent asynchrony was autotrigger, in 17/105 tested situations, followed by ineffective effort (5/105). No correction of asynchrony was observed during the period of gas administration. As Table 5 shows, and as in the bench study model, there were significant differences depending on the type of gas and ventilator model used. 
Table 5

Percentages and types of asynchronies induced by the introduction of external gas as a function of the variables controlled in the clinical model.

\begin{tabular}{|c|c|c|c|c|c|c|}
\hline & & $\begin{array}{l}\text { No } \\
\text { asynchrony }\end{array}$ & Autotriggering & $\begin{array}{l}\text { Ineffective } \\
\text { effort }\end{array}$ & Total & $\begin{array}{l}\mathrm{p} \\
\text { value* }\end{array}$ \\
\hline \multirow[t]{5}{*}{ Ventilator } & Trilogy & $19(90.5 \%)$ & $2(9.5 \%)$ & 0 & 21 & \multirow[t]{5}{*}{$<0.01$} \\
\hline & Trilogy (AutoTrak) & $21(100 \%)$ & 0 & 0 & 21 & \\
\hline & Vivo 50 & $10(47.6 \%)$ & $6(28.6 \%)$ & $5(23.8 \%)$ & 21 & \\
\hline & Astral 150 & $19(90.5 \%)$ & $2(9.5 \%)$ & 0 & 21 & \\
\hline & PB 560 & $14(66.6 \%)$ & 7 (33.3\%) & 0 & 21 & \\
\hline \multirow[t]{3}{*}{$\begin{array}{l}\text { Type of } \\
\text { gas }\end{array}$} & $\begin{array}{l}\text { Continuous gas } 4 \\
\mathrm{l} / \mathrm{min}\end{array}$ & 33 (94.2\%) & $1(2.9 \%)$ & $1(2.9 \%)$ & 35 & \multirow[t]{3}{*}{$\begin{array}{l}\mathrm{P}< \\
0.05\end{array}$} \\
\hline & $\begin{array}{l}\text { Continuous gas } 9 \\
\mathrm{l} / \mathrm{min}\end{array}$ & $28(80 \%)$ & $5(14.3 \%)$ & $2(5.7 \%)$ & 35 & \\
\hline & $\begin{array}{l}\text { Compressor at } 9 \\
1 / \mathrm{min}\end{array}$ & $22(62.8 \%)$ & 11 & 2 & 35 & \\
\hline
\end{tabular}

\section{Discussion:}

The main findings of the study are the following. In the bench study, the introduction of external gas to the circuit in NIV was associated with activation asynchronies in $36 \%$ of the situations; autotrigger was the most frequently occurring asynchrony, followed by ineffective effort. In addition, the latter showed a greater degree of spontaneous correction during gas introduction. The occurrence of asynchronies was related to the different ventilators studied, the source and flow of the gas, and lung mechanics. The results of the clinical study also corroborated a significant increase in the number of asynchronies, but in a significantly lower proportion than in the bench study. However, the conditioning factors of the asynchronies (pulsatile flow use and trigger design) were essentially the same as those found in the bench study.

The design of the activation function of the ventilators used for NIV has undergone significant improvements, always in search of a greater sensitivity (i.e., activation against decreasing efforts of the patient) and trying to preserve specificity (i.e., avoiding unwanted activations in the absence of effort). This objective has led to significant differences in design between manufacturers, which may help to explain the findings of this study. Some of the ventilators studied (Astral, Trilogy, PB 560) are equipped with flow trigger systems, which is probably the most frequently used design at present. These systems have shown greater sensitivity than the old pressure trigger models. ${ }^{19}$ However, the use of flow thresholds with fixed values for activation can cause problems in the presence of leaks if the ventilator's leakage compensation flow is higher than its activation threshold (which might be misinterpreted as patient 
effort). For this reason, flow trigger systems are often accompanied by an algorithm that can be used to automatically adjust their sensitivity as a function of leakage. ${ }^{20}$ These algorithms operate independently of the ventilator's pre-set sensitivity level, but they can lead to paradoxes such as decreased trigger sensitivity in the presence of leaks, which may eventually favour the occurrence of ineffective effort.

To compensate for these drawbacks, alternative (but more complex) designs, such as AutoTrak ${ }^{\circledR}$ (Philips Respironics), have been devised based on the crossing points that are generated by a delayed virtual waveform and superimposed (using the flow waveform method) on the native waveform ${ }^{21}$ or are derived from a calculation based on the so-called trigger energy (Breas) of the first derivative of the flow. ${ }^{22}$ In general, these more complex systems tend to be more sensitive but have a greater tendency towards automatic activation. ${ }^{21}$ These differences in design, together with the levels of activation sensitivity specific to each manufacturer, can explain the differences in the behaviour of the ventilators analysed in this study, even under basal conditions.

Another factor that generated asynchronies was the amount of gas introduced into the system. Aerosol therapy generally requires a minimum gas flow of $8 \mathrm{~L} / \mathrm{min}$. This continuous flow contributes to the pressure level that the ventilator reaches during the respiratory cycle and causes a substantial decrease in the flow administered by the ventilator itself [9]. This decrease also automatically modifies the sensitivity of the trigger in the flow models in a manner inverse to the previously described leakage model, and may favour the appearance of asynchronies. Finally, the greater number of asynchronies induced by the compressor at equal flow could be attributed to the wide fluctuations in flow generated by the device compared to the continuous flow of gas (see Fig. 1, panel A). It should be remembered that the operating mechanism of a compressor is based on the displacement of a piston on a cylinder, which generates a pulsatile flow.

Clearly, at the frequency level, the introduction of gas into the tubing cannot be compared with other events that condition the occurrence of asynchronies, such as unintentional leaks. Some authors have evaluated the ability of different ventilators to compensate for leaks ${ }^{23}$ and have concluded that certain ventilators cannot guarantee the pre-established tidal volume when involuntary leaks are introduced, a phenomenon that they attribute mainly to the development of asynchronies (autotriggering) rather than to an inaccurate estimation of the tidal volume. Other bench studies have shown that the tidal volume estimation of home ventilators can be substantially modified by the presence of continuous or random leaks. ${ }^{23,24}$

The findings of the present study have implications for clinical practice since they reflect situations (especially the use of compressors with certain ventilator models) that may require ventilator parameters to be modified in some cases.

Some limitations of this study should be taken into account. First, in both the bench and the clinical studies, the effects were examined for short, limited periods of time. Therefore, we do not know the compensation capacity of the ventilators over longer periods of time, although, based on the

Page $10 / 14$ 
recalculation algorithms against leaks at level zero, these usually occur after only a few cycles. Second, the model was applied while the patient was awake; although this is when most similar interventions are performed, the supply of oxygen in the tubing is usually delivered over the long term, and we do not know whether the systematic use of supplemental oxygen is associated with a higher percentage of asynchronies at night. Finally, to avoid premature oxidation problems with the simulator, pressurized medical gas ( $21 \%$ oxygen accompanied by $78 \%$ nitrogen) was used. Under clinical conditions, $100 \%$ pure oxygen supplementation would have been used. The different densities of pure oxygen relative to air (relative density to air 1.1052) may modify the calibration, and the ventilator's response to the flow is different from that evaluated in this study. The density $(\mathrm{g} / \mathrm{L})$ and viscosity (micropoises) of oxygen ( 1.429 and 192.6 respectively) are higher than those of air (1.293 and 170.8 respectively)

In conclusion, when an external gas is introduced into the circuit in NIV mode, the ventilators tested show different responses of asynchronies and different degrees of correction. These behaviours differ according to the amount of gas, the activation level and the external gas source. These findings make it advisable to monitor, and possibly change, the parameters when certain ventilator models and applied gas sources are used.

\section{Declarations}

\section{Ethics approval and consent to parcipate:}

The study was approved by the hospital ethics committee "Comité de ética e investigación con medicamentos (CEIm) del Parc Taulí de Sabadell" (ref. 2019/511). Written informed consent was obtained.

\section{Consent for publication}

Not applicable

\section{Availability of data and materials}

Not applicable

\section{Competing interests}

Dr Javier Sayas declares consulting fees from Philips Respironics and Resmed, and honoraria for teaching activities and participation as an advisory board member from Philips Respironics. Dr Manel Luján declares consulting fees from Breas $A G$ and honoraria for presentations from Philips Respironics and Resmed. The other authors have nothing to disclose.

\section{Funding}

Not applicable 


\section{Authors' contributions}

CL: Corresponding author. Conducting investigation process, specifically performing the experiments and data/evidence collection, statistical analysis and coordination the activity planning; execution, preparation and creation of the published work, ML and JS conceptualization and supervision: Ideas; formulation of overarching research goals and aims. Oversight of the research activity planning and execution, including mentorship external to the team and design the methodology. PF, CG, RL, and MC participation in performing the experiments, data/evidence collection and data curation.

All authors read and approve the final manuscript.

\section{Acknowledgements}

Not applicable

\section{References}

1. Rochwerg, B. et al. Official ERS/ATS clinical practice guidelines: noninvasive ventilation for acute respiratory failure.Eur Respir J2017;50.

2. Egea-Santaolalla, C. J., Chiner Vives, E., Díaz Lobato, S. \& Mangado, N. G. Lujan Tomé M, Mediano San Andrés O. Ventilación mecánica a domicilio. Open Respir Arch, 2, 67-88 (2020).

3. Rzepka-Wrona, P., Skoczynski, S., Wrona, D. \& Barczyk, A. Inhalation Techniques Used in Patients with Respiratory Failure Treated with Noninvasive Mechanical Ventilation. Can Respir J, 2018, 8959370 (2018).

4. Ehrmann, S. et al. Aerosol therapy in intensive and intermediate care units: prospective observation of 2808 critically ill patients. Intensive Care Med, 42, 192-201 (2016).

5. Jenkins, C. R. et al. Seasonality and determinants of moderate and severe COPD exacerbations in the TORCH study. Eur Respir J, 39, 38-45 (2012).

6. Peng, Y. et al. Which Nebulizer Position Should Be Avoided? An Extended Study of Aerosol Delivery and Ventilator Performance during Noninvasive Positive Pressure Ventilation. Respir Int Rev Thorac Dis, 95, 145-153 (2018).

7. Saeed, H. et al. Modeling and optimization of nebulizers' performance in non-invasive ventilation using different fill volumes: Comparative study between vibrating mesh and jet nebulizers. Pulm Pharmacol Ther, 50, 62-71 (2018).

8. Hess, D. R. Aerosol Therapy During Noninvasive Ventilation or High-Flow Nasal Cannula. Respir Care, 60, 880-891 discussion 891-893. (2015).

9. Schwartz, A. R., Kacmarek, R. M. \& Hess, D. R. Factors affecting oxygen delivery with bi-level positive airway pressure. Respir Care, 49, 270-275 (2004).

10. Ari, A. \& Fink, J. B. Differential Medical Aerosol Device and Interface Selection in Patients during Spontaneous, Conventional Mechanical and Noninvasive Ventilation. J Aerosol Med Pulm Drug Deliv, 
29, 95-106 (2016).

11. Hassan, A., Salah Eldin, R., Abdelrahman, M. M. \& Abdelrahim, M. E. In-vitro/in-vivo comparison of inhaled salbutamol dose delivered by jet nebulizer, vibrating mesh nebulizer and metered dose inhaler with spacer during non-invasive ventilation. Exp Lung Res, 43, 19-28 (2017).

12. Dhand, R. Aerosol delivery during mechanical ventilation: from basic techniques to new devices. $J$ Aerosol Med Pulm Drug Deliv, 21, 45-60 (2008).

13. Fauroux, B., Leroux, K., Pépin, J-L., Lofaso, F. \& Louis, B. Are home ventilators able to guarantee a minimal tidal volume? Intensive Care Med, 36, 1008-1014 (2010).

14. Mullender, M. et al. A Dutch guideline for the treatment of scoliosis in neuromuscular disorders. Scoliosis, 3, 14 (2008).

15. Ehrmann, S. et al. Ventilator-integrated jet nebulization systems: tidal volume control and efficiency of synchronization. Respir Care, 59, 1508-1516 (2014).

16. Rello, J. et al. Key considerations on nebulization of antimicrobial agents to mechanically ventilated patients. Clin Microbiol Infect Off Publ Eur Soc Clin Microbiol Infect Dis, 23, 640-646 (2017).

17. Olivieri, C., Costa, R., Conti, G. \& Navalesi, P. Bench studies evaluating devices for non-invasive ventilation: critical analysis and future perspectives. Intensive Care Med, 38, 160-167 (2012).

18. Gonzalez-Bermejo, J. et al. Framework for patient-ventilator asynchrony during long-term noninvasive ventilation., 74, 715-717 (2019).

19. Aslanian, P. et al. Effects of flow triggering on breathing effort during partial ventilatory support. Am J Respir Crit Care Med, 157, 135-143 (1998).

20. Innovation and technology | ResMed n.d. https://www.resmed.com/ap/en/healthcareprofessional/products/innovation-and-technology/ventilation-innovation-and-technology.html (accessed July 31, 2020).

21. Prinianakis, G., Kondili, E. \& Georgopoulos, D. Effects of the flow waveform method of triggering and cycling on patient-ventilator interaction during pressure support. Intensive Care Med, 29, 1950-1959 (2003).

22. US Patent Application for A SYSTEM AND METHOD FOR SYNCHRONIZATION OF BREATHING IN A MECHANICAL VENTILATOR Patent Application (Application \#20160008559 issued January 14, 2016) - Justia Patents Search n.d. https://patents.justia.com/patent/20160008559 (accessed July $31,2020)$.

23. Luján, M. et al. Influence of dynamic leaks in volume-targeted pressure support noninvasive ventilation: a bench study. Respir Care, 60, 191-200 (2015).

24. Luján, M. et al. Effect of leak and breathing pattern on the accuracy of tidal volume estimation by commercial home ventilators: a bench study. Respir Care, 58, 770-777 (2013).

\section{Figures}




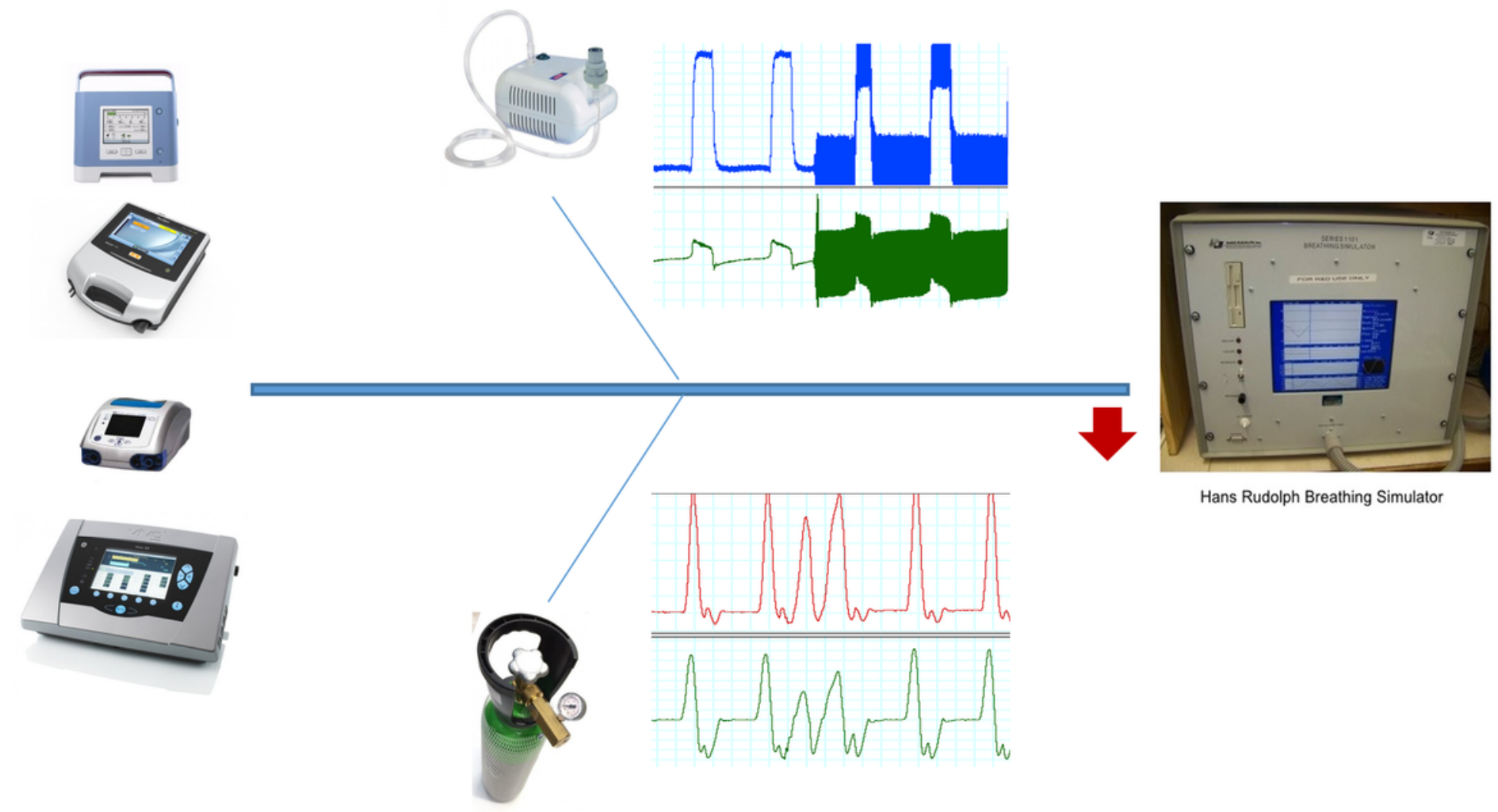

Figure 1

The flow of the compressor was monitored prior to placement, and a value of $9 \mathrm{l} / \mathrm{min}$ was obtained 